\title{
Mit der Peitsche in den dreckigen Orient: Die Darstellung des Orients in Oskar Manns Reisebriefen
}

\section{With the Whip into the Dirty Orient: The Depiction of the Orient in Oskar Mann's Travel Letters}

\author{
Remzi $\mathrm{AVCl}^{1}$ (D)
}

'Dr. Ögretim Üyesi, Mardin Artuklu Üniversitesi, Tarih Bölümü, Mardin, Türkiye

ORCID: R.A. 0000-0002-8539-9203

\section{Corresponding author:}

Remzi AVCl,

Mardin Artuklu Üniversitesi, Tarih Bölümü, Mardin, Türkiye

Email: remziavci@gmail.com

Submitted: 30.08 .2021 Accepted: 08.10.2021

Citation: Avci, R. (2021). With the whip into the dirty orient: The depiction of the orient in Oskar Mann's travel letters. Alman Dili ve Edebiyatı Dergisi - Studien zur deutschen Sprache und Literatur, 46, 101-119. https://doi.org/10.26650/sds|2021-988686

\begin{abstract}
DEUTSCH)
Der vorliegende Beitrag thematisiert die Reisebriefe des deutschen Orientalisten Oskar Mann (1867-1917). Mit finanzieller Unterstützung der Königlich-Preußischen Akademie der Wissenschaften unternahm Mann zwischen 1901 und 1906 zwei große wissenschaftliche Reisen in den Osten des Osmanischen Reiches und in den Iran, um die iranischen Sprachen und Dialekte zu erforschen. Die Reisebriefe und -tagebücher sind Texte mit relativ subjektiven Werturteilen, in denen häufig Menschen und Kulturen durch ethnozentrische Stereotype beschrieben wurden, da die realen Reisen eine kulturelle Begegnung und Konfrontation mit dem Anderen darstellen. Die Beschreibung einer fremden Kultur kann nicht von den subjektiven Werturteilen des Reisenden getrennt werden. Das heißt, die fremde Welt, in der sich der Reisende bewegt, ist die Projektion der äußeren Umgebung durch subjektive Wahrnehmung. Nach seiner Ansicht handelt es sich bei den Orientalen um Menschen eines Ortes, der sich dem Westen ergeben hatte. Mann trennt den Orient vom Okzident mit präzisen und scharfen Linien und teilt eurozentristisch in zwei voneinander getrennte Kategorien. Während seiner Reise produzierte und vermittelte Mann einerseits Wissen über fremde Kulturen, andererseits verbreitete und verstärkte er Bilder und Vorurteile sowie auch Stereotype, die zu ontologischen Unterscheidungen zwischen Orient und Okzident führten. Dieser Aufsatz versucht zu zeigen, dass Mann den Orient mit hegemonialem Denkmustern wahrgenommen hat; und dass seine Fremdvorstellung zutiefst dem klassisch europäischen orientalistischen Diskurs des 19. Jahrhunderts verhaftet bleibt, was zur Folge hat, dass der Orient abgewertet wird. Es wird in Frage gestellt, mit welchen Stereotypen, Bildern und Vorstellungsmustern Mann die Bevölkerung des bereisten fremden Landes darstellt.
\end{abstract}

Schlüsselwörter: Oskar Mann, Iran, iranische ethnische Gruppen, Orientvorstellung, Reisebriefe, Stereotype

\section{ABSTRACT (ENGLISH)}

The present article deals with the travel letters of the German orientalist Oskar Mann (1867-1917). With financial support from the Royal Prussian Academy of Sciences, Mann made two expeditions to the Ottoman Empire and Iran between 1901 and 1906 to research the Iranian languages and dialects. Travel letters and travel diaries 
are texts with relatively subjective value judgments, in which people and cultures are often described using ethnocentric stereotypes, because a real journey represents a cultural encounter and confrontation with the other that offers unique and invaluable information about the new world. The description of a foreign culture cannot be separated from the subjective value judgments of a traveller. This means the foreign world in which the traveller moves is represented by the subject who experiences it. According to Mann, the Orientals are people from a place that has surrendered to the West. He separates the Orient from the Occident with precise and sharp lines and divides them Eurocentrically into two separate categories. During his travels, Mann produced and imparted knowledge about the foreign cultures on the one hand, and on the other hand he spread and reinforced images and prejudices as well as stereotypes that led to the ontological differentiation between Orient and Occident. This essay tries to show that he perceived the Orient with hegemonic thought patterns and that his foreign imagination remained deeply rooted in the classic European orientalist discourse of the 19th century, and as a consequence the Orient was devalued. This study discusses the stereotypes, images and pattern of ideas that he used to represent the population of the foreign country where he travelled.

Keywords: Oskar Mann, Iran, Iranian ethnic groups, Orient-Imagination, travel letters, stereotypes

\section{EXTENDED ABSTRACT}

Over the course of time, people from different professions left behind travel books or letters which were not put into book form. In the $19^{\text {th }}$ century, travels to the Orient found their expression in travel literature, which could be of a scientific, fictional, military or diplomatic nature. Edward Said's book Orientalism is of great importance in understanding the Western form of perception of the Other, by combining detailed text interpretations with discourse analysis. Said has made a significant contribution to making this relationship visible. According to his interpretation, "the Orient is an imaginary geography and space construed by the ideology, the cultural set of values and norms of the West". He also deals with the question of how travel reports constitute this invented Orient. Travel letters and travel diaries are texts with relatively subjective value judgments, in which people and cultures are often described using ethnocentric stereotypes, because a real journey represents a cultural encounter and confrontation with the other that offers unique and invaluable information about the new world. The European travellers create, disseminate and enhance images, prejudices and stereotypes that lead to the ontological differentiation between Orient and Occident. The German Iranologist Oskar Mann studied oriental philology (Sanskrit, Arabic, Aramaic and comparative linguistics) between 1886 and 1891 in Heidelberg and Berlin and in 1890, received his doctorate in Strasbourg under Theodor Nöldeke (1836-1930). Between 1901 and 1906, Mann accomplished two great journeys to the Ottoman Empire and Iran to study the Iranian languages and dialects. While his first expedition through Iran in 1901 lasted two years, the second travel to the Ottoman Empire in 1906 lasted only one year. Both of his trips within the Orient were financed by the Königlich-Preußischen Akademie der Wissenschaften (PrussianKingdom Academy of Sciences). Kaiser Wilhelm II was very interested in Mann's field 
research and gave him financial support in the amount of 30,000 marks during his trip. From 1901 to 1903, Mann crossed Iran, starting from Bushehr in the northwest, and visited its most important cities, such as Shiraz, Isfahan, Kirmanschah and Khoramabad, among others. His second journey, this time to the Ottoman Empire, began on January 27, 1906 with a steamboat from Hamburg to Alexandria and Beirut. From there he reached Aleppo. He then crossed the eastern part of the empire on horseback in the direction of Birecik-Urfa-Siverek-Diyarbakır-Palu-Muş-Bitlis-Cizre and Baghdad. On both of these trips, he conducted research on the Iranian languages and dialects and collected philological material on them. In terms of content, Mann's letters can be categorized in two ways: first, letters written in diary form to his family, and second, scientific letters and reports that contain detailed information on his philological research. His letters have a narrative and literary character. He describes the exact course of his travel and informs the reader about many details: people, cultures, nature, the weather, and the topography and geography of the Orient.

Mann felt obliged to write down his subjective impressions as well as to collect scientific-philological information based on his consciousness of his audience. This moved him to emphasize certain aspects and striking peculiarities of the foreign countries that would resonate in his own nation. In addition, the life story, the personal environment, the professional competence, the cultural thought patterns, and the intentions he pursues with his text shape the traveller's perception, which is directly reflected in his texts. The description of a foreign culture cannot be separated from the subjective value judgments of a traveller. This means the foreign world - in which the traveller moves - is represented by the subject who experiences it. In Mann's opinion, the Orientals are people from a place that has surrendered to the West. He separates the Orient from the Occident into two separate categories with precise and sharp lines. Compared to European culture, the Orient appears in his letters to be underdeveloped, dirty, and barbaric. He produces certain stereotypes about the different Iranian ethnic groups and regards them as inferior. In order to legitimize his descriptions and judgments based on negative stereotypes, Mann speaks of his own experiences, which he has gained on Iranian and Ottoman soil. It is indeed this memory that inspires Mann's daring attempt to try to tame the Orientals with a whip in hand. 


\section{Einleitung}

Im Laufe der Zeit hinterließen Menschen aus verschiedenen Berufsgruppen Reisebücher oder auch Reisebriefe, die nicht in Buchform gebracht wurden. Im 19. Jahrhundert fanden Orientreisen ihren Niederschlag in Reiseliteratur, die wissenschaftlicher, belletristischer, militärischer, diplomatischer Natur sein konnte oder sich als Fantasy und Fiktion gehörte. Bei der Vielzahl der Gattungen ist es schwer, Reisetexte als einheitliches Genre' zu kategorisieren und zu systematisieren (vgl. Wagner, 2016, S. 80). Es ist daher ratsam, die Verschriftlichung der Reisenden selbst zu beschreiben. Die Übertragungs- oder Darstellungsform von Reiseeindrücken hängt von vielen Faktoren ab wie dem sozialen Status, Bildungshintergrund, Vorwissen der Wahrnehmungsfähigkeit und Neugier des Reisenden (vgl. Wagner, 2014, S. 99). Oskar Manns Reisebriefe, die Gegenstand dieser Arbeit sind, können als Reisetagebücher klassifiziert werden, die auf der „erzählenden Darstellung einer realen Reise“ basieren (Brenner, 1990, S. 1). Die Reiseindrücke wurden vom Reisenden hauptsächlich als Buch publiziert. Daher erfordern unveröffentlichte Briefe, die während der Reise geschrieben wurden, eine unterschiedliche Klassifizierung, da sie im Vergleich zu veröffentlichen Reisetexten wie Büchern, Aufsätzen, Berichten usw. mehr authentische, natürliche und präzise Werturteile und Darstellungen enthalten.

Edward Saids (1979) Buch Orientalism hat große Bedeutung für das Verständnis der westlichen Wahrnehmungsform des orientalistischen Fremden, indem es detaillierte Textinterpretationen mit Diskursanalysen kombiniert. Said argumentiert, dass die westliche Zivilisation den Orient als den Anderen, als ihr Gegenbild und ihre Gegenidee geschaffen hat, indem sie den Orient institutionell, edukativ und linguistisch, aber auch mit Prinzipien, sogar strukturell in Bezug auf die Kolonialverwaltung unterstützend in eine neue Entität und Form verwandelte (vgl. Said, 1979, S. 1-2). Der Orientalismus und Reisen sind eng miteinander verbunden. Said hat maßgeblich dazu beigetragen, diese Beziehung sichtbar zu machen. Gemäß seiner Interpretation ist der Orient ein imaginärer Ort. Dabei beschäftigt er sich auch mit der Frage, wie die Reiseberichte diesen erfundenen Orient konstituieren. Nach der Veröffentlichung von Orientalism wurden die weiteren Studien unternommen, die Reiseberichte unter einem neuen und kritischen Blickwinkel betrachten. Die europäischen Orient-Repräsentationen sind in den letzten Jahrzehnten

1 Es gibt zahlreiche Literatur über die Gattung Reisebericht. Bei der Kategorisierung und Systematisierung von Reiseberichten haben die Werke von Peter J. Brenners (1990), Veruscka Wagner (2016) und Tanja Hupfeld (2007) Zouheir Soukah (2016) sowohl theoretisch als auch methodologisch inspiriert. 
ein wichtiges Forschungsthema in den Geistes- und Kulturwissenschaften geworden. Dafür bilden die Reiseberichte ein bemerkenswertes Quellengenre (vgl. Gogiaschwili, 2008, S. 253). Die Reisebriefe und -tagebücher sind Texte mit relativ subjektiven Werturteilen, in denen häufig Menschen und Kulturen durch ethnozentrische Stereotype beschrieben wurden, da die realen Reisen eine kulturelle Begegnung und Konfrontation mit dem Anderen darstellen. Wie auch Zouheir Soukah (2016) in seiner Dissertation betont, gibt es jetzt spezielle Studien, ${ }^{2}$ die die Orientwahrnehmung von Reisenden nur in bestimmten Ländern wie Marokko, Tunesien, Ägypten und Jemen untersuchen.

Einerseits produzieren und vermitteln die europäischen Reisenden großen Wissensvorteil über fremde kulturelle Gemeinschaften oder Bevölkerungsgruppen, noch nie gesehenen Naturerscheinungen oder unerforschten Orte (vgl. Bies und Košenina, 2014, S. 7), anderseits erzeugen, verbreiten und verstärken sie Bilder, Vorurteile und Stereotype, die zur ontologischen Unterscheidung zwischen Orient und Okzident führen. Obwohl die Begriffe Stereotyp, Klischee und Vorurteil alltagssprachlich Synonyme darstellen, werden diese in den Sozialwissenschaften differenziert verwendet. Bei Stereotypen, die auf emotionalen Bewertungen beruhen und dementsprechend keine objektiven Wahrnehmungen sind, handelt es sich um „Bilder in unseren Köpfen“, die als „unveränderbar und überzeitlich“ gelten. Vorurteile sind hingegen stabile negative Einstellungen gegenüber einer Person oder Gruppe und basieren gemeinhin nicht auf individuellen Erfahrungen. Im Gegensatz zu Vorurteilen, die meist abwertend sind, beruhen Stereotype auf Erlebnissen und sind nicht immer negativ (Wagner, 2016, S. 202203). Stereotype Bilder, Urteile, Wahrnehmungen und Vorstellungen können auf existenten Gegebenheiten fußen, andererseits können sie zu einem erheblichen Teil aus irrealen Bestandteilen bestehen. Darüber hinaus sind Stereotype strenge Verallgemeinerungen, Vereinfachungen und Kategorisierungen (vgl. Voss, 2001, S. 254; vgl. Wagner, 2016, S. 203; vgl. Voltrova, 2010, S. 140).

Oskar Manns Briefe wurden bisher in wenigen philologischen Einzelstudien nur zum Teil untersucht und aus historischer Sicht in einer Einzelarbeit nicht vollständig behandelt. Die philologischen Studien von Mann waren kürzlich Gegenstand eines Artikels (Avcl, 2021). Die erwähnte Arbeit untersucht inn ausschließlich als philologischer Wissenschaftler. Die vorliegende Abhandlung analysiert die Briefe basierend auf der

2 Khalid Lazaare (1998) und Mounir Fendri (1996) konzentrieren sich auf die Beschreibung und Darstellung der deutschen Reisenden in einer islamischen Gesellschaft im Fall von Nordafrika. Beispiele für Studien, die sich auf eine bestimmte islamisch-orientalische Region konzentrieren, können erweitert werden. 
Arbeit Persische und kurdische Reiseberichte, die Motjaba Kolivand edierte und als Buch veröffentlichte (2014). Die im Oskar Mann-Nachlass befindlichen Briefe wurden von Kolivand als Persische Reisebriefe (erste Reise 1901-1903) und kurdische Reisebriefe (zweite Reise 1906-1907) chronologisch klassifiziert und nummeriert. Er schließt mit diesem Buch eine wichtige Lücke in der bisherigen Literatur zu Oskar Mann. Auf seinen beiden Reisen schrieb Mann Briefe an seinen Kollegen, den Iranisten Friedrich Carl Andreas (1846-1930), den Orientalisten Eduard Sachau (1845-1930) und seine Schwester Marthe Mann. Darüber hinaus sandte er einige Reiseberichte an die Königlich-Preußische Akademie der Wissenschaften, in denen er sich über seine philologischen Forschungen an den iranischen Dialekten und Sprachen ausließ (vgl. Kolivand 2014, S. 9-15). ${ }^{3}$

Mit der Institutionalisierung der orientalischen Philologie im 19. Jahrhundert an europäischen Universitäten nahm das wissenschaftliche Interesse an der außereuropäischen Welt zu (vgl. Osterhammel, 1989a, S. 14). Manns Reisen waren in erster Linie eine wissenschaftlich-praktische Fortsetzung dieses Interesses. Seine Reisebriefe geben sehr umfangreiche Informationen über diverse konfessionell-religiöse, ethnische Volksgruppen mit verschiedenen Sprachen als auch Dialekten sowie über Mann selbst und über die Gegebenheiten während seiner Exkursion. Dieser Aufsatz sucht zu zeigen, dass Mann die orientalisch-iranischen Volksgruppen mit bestimmten stereotypen Denkmustern repräsentierte; und dass seine Fremdvorstellung zutiefst dem klassisch-europäischen orientalistischen Diskurs des 19. Jahrhunderts verhaftet bleibt und er einen abwertenden Orient konstituiert. Dazu ist es nötig festzustellen, mit welchen Stereotypen, Bildern und Vorstellungsmustern Mann die Bevölkerung des bereisten fremden Landes darstellt.

Die Argumentation gliedert sich in drei Hauptteile. Der erste Teil untersucht Manns Biografie, den Verlauf und die wesentlichen Merkmale/Aspekte seiner Reise. Der zweite Teil beschäftigt sich mit der Frage, wie Mann die orientalischen Volksgruppen und den Orient als Raum ausführlich wahrnimmt, darstellt und übermittelt. Der dritte Teil diskutiert, wie Mann sich bei seinen Beobachtungen der Menschen auf einige pauschale Urteile beschränkt.

3 Die von Kolivand bearbeiteten Briefe befinden sich in drei verschiedenen Archiven in Deutschland. 1) Handschriftenabteilung der Staatsbibliothek zu Berlin (Briefe an Familie) 2) Niedersächsische Staats- und Universitätsbibliothek Göttingen (Briefe an C. F. Andreas) 3) Archiv der Berlin-Brandenburgischen Akademie der Wissenschaften (Briefe an die Königlich-Preußischen Akademie der Wissenschaften). 


\section{Oskar Mann: Philologe, Reisender, Forscher und Beobachter}

Oskar Mann wurde am 18. September 1867 in Berlin geboren. Seit seiner Kindheit interessierte er sich für Geschichte und Sprachen. Zwischen 1886 und 1891 studierte er Orientalische Philologie (Sanskrit, Arabisch, Aramäisch und vergleichende Sprachwissenschaft) in Heidelberg und Berlin. Darüber hinaus nahm er Privatunterricht bei den Orientalisten Carl Friedrich Andreas und Friedrich Rosen (1856-1935) über die persische Sprache, Kultur, Geografie und Geschichte (vgl. Kolivand, 2014, S. 9). Mann promovierte 1890 in Straßburg beim bekannten Orientalisten Theodor Nöldeke (18361930). In seiner Dissertation bearbeitete und übersetzte er eine persische Handschrift über die Geschichte und Kultur des Iran zwischen 1747 und 1750 von Abu'I-Hasan IbnMuhammad. Er veröffentlichte sie dann unter dem Titel Das Mujmil et tarikh-i ba'dnadirije des Ibn Muhammed Emin Abu'I-Hasan aus Golistane (Mann 1891). Nach seiner Promotion begann er eine Laufbahn an der Königlichen Bibliothek zu Berlin und wurde später Leiter der orientalischen Handschriftenabteilung. Mann arbeitete mit Unterbrechungen bis zu seinem Tod 1917 als Bibliothekar (vgl. Kolivand, 2014, S. 9; vgl. Teilhaber 2020, S. 125).

Manns Orientreisen wurden von der Königlich-Preußischen Akademie der Wissenschaften finanziert (vgl. Mann, 1906, S. VIII-IX). Kaiser Wilhelm II. war an Manns Forschungen sehr interessiert und gab ihm während seiner Reise eine finanzielle Unterstützung in Höhe von 30.000 Mark (vgl. Lemke und Rosbeiani 2018, S. 26). Wie Friedrich Althoff Kaiser Wilhelm II. berichtete, war die Bagdadbahn-Konzession ziemlich interessiert an Manns Arbeit über kurdische Dialekte, da sich einige Strecken der Bagdadbahn-Linie durch kurdisches Gebiet erstreckten. Trotz des Interesses finanzierte die Konzession Manns Reisen nicht (vgl. Marchand, 2009, S. 338).

Mann begann seine erste Reise am 20. Oktober 1901 mit dem Zug nach Marseille. Von dort fuhr er mit dem Dampfschiff nach Port Said und Bombay. Nach vier Wochen erreichte er die Stadt Buschehr am Persischen Golf (vgl. Kolivand 2014, S. 10). Er durchquerte von 1901 bis 1903 den Iran von Buschehr im Nordwesten und besuchte dessen wichtigste Städte wie Schiraz, Isfahan, Kirmanschah und Khoramabad usw. Mit der Entwicklung der Weltverkehr wie Eisenbahn und Dampfschiff, Telegraphie und Post im 19. Jh. hatte sich die kulturelle Praxis des Reisens grundlegend verwandelt. Neben der Mobilität spielten auch die wissenschaftlichen Entwicklungen in der Geographie und Kartographie eine wichtige Rolle bei der Zunahme der Reisen in den Orient in dieser Epoche. Die Ausbreitung des Kolonialismus ermöglichte es den europäischen Bürgern, sich freier und 
bequemer zu bewegen (vgl. Avcl, 2020, s. 32). Neben philologischen Forschungen dokumentierte Mann die elamitischen Inschriften und andere archäologische Denkmäler (vgl. Mann, 1903, S. 327-331; vgl. Huff, 2001).

Manns zweite Reise in das Osmanische Reich begann am 27. Januar 1906 mit einem Dampfschiff von Hamburg nach Alexandria und Beirut. Von dort erreichte er Aleppo. Anschließend durchquerte er zu Pferd den Osten des Reiches in Richtung Birecik-UrfaSiverek-Diyarbakır-Palu-Muş-Bitlis-Cizre und Bagdad. Bei dieser Reise unternahm er philologische Feldforschungen über die kurdische und die Zaza-Sprache (vgl. Kolivand 2014, S. 11). Während seiner beiden Reisen sammelte Mann eine große Menge philologischer Materialien über iranische Sprachgruppen wie Persisch, Kurdisch, Zaza, Gorani, Luri, Mukri, Tajik usw. Nach der Rückkehr nach Berlin veröffentlichte er die Ergebnisse seiner Expeditionen unter dem Titel Die Mundart der Mukri-Kurden (Mann, 1906), das erste Buch der Reihe Kurdisch Persische Forschungen (vgl. Paul 2020, S. 290). Darüber hinaus veröffentlichte er 1909 Die Tâjîk-Mundarten der Provinz Fârs (Mann, 1909) als Fortsetzung der Reihe und nach einem Jahr Die Mundarten der Lur-Stämme im südwestlichen Persien (Mann, 1910). Nach Manns Tod wurde der Orientalist Karl Hadank (1882 - 1945) von 1919 bis 1945 von der Akademie der Wissenschaften beauftragt, die sich in Manns Nachlass befindenden unverarbeiteten Materialien zu klassifizieren und zur Veröffentlichung vorzubereiten (vgl. Paul 2020, S. 293).

Inhaltlich können Manns Briefe auf zwei Arten kategorisiert werden. Erstens: Briefe an die Familie, die im Tagebuchformat geschrieben sind. Zweitens: Die wissenschaftlichen Brief-Berichte, die über den Inhalt der philologischen Forschungen ausführliche Informationen enthalten. In den Briefen beschreibt Mann seine Gefühle und Eindrücke mit einem eigenen einzigartigen Stil, also mit einer ungewöhnlichen Ausdrucksweise, in einer lustigen, umgangssprachlichen und fließenden Sprache. Seine Briefe haben einen narrativ-detaillierten Charakter. Er schildert den genauen Ablauf seiner Reise chronologisch und informiert seinen Leser nicht nur über seine philologischen Forschungen, sondern auch über viele Einzelheiten: Menschen, Kulturen, die Natur, das Wetter, die Topografie und Geografie des bereisten Orients. Manns deskriptive Erzählkraft verstärkt die Informations- und Wahrnehmungsvermittlung in seinen Briefen. Wie Günter Funke (vgl. 1982, S. 97) betont, die individuellen Erlebnisse und Analyse des Reisenden legen das Format des Reisetexts fest. In diesem Zusammenhang kann man sagen, dass die reichlichen Erfahrungen, die Mann auf seinen langen Reisen sammelte, sich in seiner deskriptiven Darstellung niederschlagen. 
Mann war immer mit seinen Dienern und Reiseführern unterwegs. Sowohl seine enge Beziehung zu den lokalen Verwaltern und Würdenträgern der Region als auch seine Beherrschung der iranischen Sprachen und Dialekte spielten eine wichtige Rolle bei seiner Mobilität. Die einheimischen Notabeln verwendet er für seine Sicherheit. Dies spiegelt sich in einem Brief von Kermanschah wie folgt wider: „Doch ist er zugleich Mirza und Dolmetscher [...] auch gewissermassen eine Art Schutz für mich gegen seine Landleute" (Brief an Eduard Sachau vom 6 Juli 1902, Kolivand, S. 178). Manns Orient ist kein sicherer Ort. Daher fühlt er sich nie zuversichtlich, dass er allein, ohne Waffen und ohne Aufsicht der lokalen Mächte reisen könnte: „[...] dann verschiedene Ausflüge in die nähere und weitere Umgegend in das Gebiet der einzelnen Kurdenstämme - Alles ohne Gefahr, da ich mit den Stammesoberhäuptern dicke Freundschaft geschlossen habe, und die guten Leuten alles Mögliche für mich thun“ (Brief an die Familie vom 21 Juli 1902, Kolivand, S. 183).

\section{Die Wahrnehmung und Vermittlung des Orients}

Die Reiseliteratur des 19. Jahrhunderts kann auch als kulturelles Produkt des Imperialismus angesehen werden. In der ersten Hälfte dieses Jahrhunderts entstanden vielfältige landeskundliche Werke aus den Federn britischer Kolonialfunktionäre. Bei diesen handelt es sich regelmäßig um Beamte der East Indian Company. Während des Aufenthalts in den Kolonien verfassten diese Männer Reiseberichte oder -briefe (gedruckt oder ungedruckt), die zur Institutionalisierung des Kolonialismus beitrugen oder die notwendigen Informationen zum Aufbau der Infrastruktur des Kolonialismus lieferten. Diesem Muster folgten dann andere kolonial-europäische Mächte. Schließlich nahmen die Reisetexte verschiedener Gattungen, insbesondere in der zweiten Hälfte des 19. Jahrhunderts, erheblich zu, da die große Mehrzahl der zuvor in den Orient gereisten Europäer Seeleute, Diplomaten oder Soldaten waren. Diese Männer notierten ihre Beobachtungen und Erlebnisse ganz selten (vgl. Osterhammel, 1989a, S. 12-23). Eine Anzahl von wissenschaftlichen Reisen wurde im 19. Jahrhundert von offiziellen oder halboffiziellen Regierungsbehörden finanziert (vgl. Edwards und Rune, 2011, S. 11). Auf Grundlage einer gesamteuropäischen Kolonialpolitik und Verflechtungen betrachtet, stellen die deutschen Reisetexte im späten 19. und frühen 20. Jahrhundert ein Nebenprodukt dar (Osterhammel, 1989b, S. 229). Warum unterstützte das junge Deutsche Reich Manns philologische Reise in den Orient? Die Antwort auf dieser Frage ist eng mit der kolonialpolitischen Orientierung Deutschlands verknüpft, weil das Deutsche Reich seinen politischen Einfluss im osmanisch-iranischen Raum durch die 
friedliche Durchdringung erweitern wollte. Die wissenschaftlichen Expeditionen in diesen Regionen können ebenfalls als Teil der deutschen Orientpolitik betrachtet werden.

Hinter Manns Reise steht eine ähnliche Motivation. Auch sie basiert auf einer hegemonialpolitischen Macht seines kolonialen Reiches, obwohl er zu wissenschaftlichen Zwecken in eine fremde Welt aufbrach. Er fühlte sich verpflichtet, seine subjektiven Eindrücke sowie gesammelten wissenschaftlich-philologischen Informationen in Überzeugung eines gezielten Adressatenkreises (in Manns Fall vor allem Verwandte, Berufskollegen und Institutionen) niederzuschreiben. Dies beeinflusste seine Wahrnehmung und bewegte ihn, bestimmte Aspekte und auffallende Besonderheiten des bereisten fremden Landes - des Orients - hervorzuheben, die Resonanzen in der eigenen Nation finden würden (vgl. Kabbani, 1986, S. 1). Es sind die Biografie, die privaten und beruflichen Erfahrungen des Reisenden, epochale und kulturelle Ideologien, seine europäisch geprägten Erwartungen sowie das Ziel seiner Veröffentlichung, die die Wahrnehmung einer fremden Kultur während der Reise beeinflussen (vgl. Hupfeld, 2007, S. 27). Dadurch spiegeln sich die Wahrnehmungsmuster des Reisenden direkt im Text wider, denn die Beschreibung einer fremden Kultur kann nicht von den subjektiven Werturteilen des Reisenden getrennt werden. Das heißt, die fremde Welt, in der sich der Reisende bewegt, ist die Projektion der äußeren Umgebung durch subjektive Wahrnehmung (vgl. Wagner, 2016, S. 84). Während seiner Reise konstruiert Mann in seinen Briefen ein sehr abwertendes Bild der orientalisch-islamischen Kultur im Iran und im östlichen Teil des Osmanischen Reiches, das auf festgelegten Wahrnehmungsmustern beruht. Manns Orientvorstellung speist sich aus einer Unbekanntheit der Örtlichkeit und einem subjektiven kritischen Vergleich mit der westlichen Zivilisation. Sein Orient - eine in der Realität ihm sehr unbekannte Welt - begrenzte sich auf das, was er in Büchern las und hörte.

Die Darstellung der fremden Außenwelt in Reiseberichten, unterliegt so a priori einer kulturell hervorgebrachten Selbstkonstruktion (vgl. Wagner 2016, S. 84). Während seiner beiden Reisen beschreibt Mann die Sitten, Bräuche, die Kultur und die Menschen des zeitgenössischen Orients, indem er ihn mit dem Raum vergleicht, von dem er gekommen ist. Mann zieht eine scharfe ethnozentrische Grenze zwischen Europäern und orientalischen Menschen. Er verwendet das Wort Kerl, wenn er über einen orientalischen Menschen im bereisten Land spricht. Seine einheimischen Diener, die er öfter als Kerls bezeichnet, werden beleidigt, selbst wenn sie pünktlich zur Arbeit kommen (Briefe an die 
Familie von 9 und 20 Dezember 1901, Kolivand, S. 44, 55); 3 Januar 1902, Kolivand, S. 59); 3 Februar 1902, Kolivand, S. 73); 16 Mai 1902, Kolivand, S. 136); 31 Mai 1903, Kolivand, S. 370-371). Im Gegensatz dazu bezeichnet er die im Orient lebenden Europäer als Herren, denn er empfindet sich dort unter den Orientalen als Teil einer herrschenden Zivilisation. Nach seiner Ansicht handelt es sich bei den Orientalen um Menschen eines Ortes, der sich dem Westen ergeben hatte und noch ergab. Es war tatsächlich dieses Gedächtnis, das Manns kühnen Wagemut anregt, die Orientalen mit einer Peitsche in der Hand zähmen zu wollen. Wie Said feststellt, "ein Engländer, der in Indien oder Ägypten im späten neunzehnten Jahrhundert lebte und sich für Indien oder Ägypten interessierte, vergaß keinen Moment, dass es britische Kolonien waren" (1979, S. 11). Mit dieser Said'schen Perspektive bildet die hegemonial-kolonial europäische Herrschaft die Grenze von Manns abwertenden Beschreibungen des Orients. Mann tritt den Menschen im Orient zumal als Europäer mit entsprechenden militärisch, wirtschaftlich und vor allem kulturellen Privilegien gegenüber und beobachtet sie mit dieser Identität (Said, 1979, s. 156). Seine Männer auf dem Weg waren nicht Freunde, sondern seine Diener. Mann betont bewusst, dass sie seine Diener sind, wenn er von ihnen in den Briefen spricht, und erinnert sie während der Reise daran, wer sie sind, mit Beschimpfungen und Auspeitschung.

Das gab großen Krach mit dem Maultiertreiber, der erst zu meinen Gunsten entschieden wurde, nachdem der Lump eine Tracht Prügel bezogen hatte. Dabei ging mein Peitschenstiel an dem harten Schädel des Kerls in tausend Stücke. Der „edele Teil" hatte aber keinen Schaden gelitten. (Brief an die Familie vom 28 September 1906, Kolivand, S. 554)

Der orientalische Diskurs und seine Traditionalisierung im Laufe der Zeit veranlasste die Europäer, den Orient mit bestimmten unveränderlichen kulturellen Mustern, Klischees und Stereotypen wahrzunehmen (vgl. Said 1979, S. 42). Diese Haltung ist in Manns Praxis deutlich zu erkennen. Während seiner Reise sperrt er den Orient in die ethnischen Stereotypen ein. Das grundlegendste Merkmal ethnisch-nationaler Stereotypen besteht darin, in der zeitlichen und räumlichen Verfremdung relevanter Aspekte (vgl. Wagner 2016, S. 203). Diese konstruieren abgestumpfte Wahrnehmungen über eine Bevölkerung oder Volksgruppe. Was von einem durchschnittlichen orientalischen Menschen in diesem Sinne erwartet wird, ist, dass er sich verspätet, faul und dreckig sein wird. Wenn man, wie Hentsch konstatiert, einige Reiseberichte aus dem 17. Jahrhundert gelesen hat, sieht es so aus, als hätte man Dutzende davon 
gelesen (vgl. 2008, S. 21). Er meint damit, dass die Reisenden ihre Eindrücke in unterschiedlichen Textarten regelmäßig in stereotypen Wendungen schildern und vermitteln. Manns Gedächtnis ist voll von diesen tradierten Verallgemeinerungen und menschenverachtenden Vorstellungen. Der entscheidende Unterschied in seinen Briefen besteht darin, dass er nicht berücksichtigte, dass seine Briefe eines Tages veröffentlicht werden würden. Deshalb verachtet und beleidigt er verschiedene Volksgruppen wie die Iraner, Kurden und Armenier. Er beschreibt Perser als die Menschen, "die nur von Lügen leben“" (Brief an die Familie vom 23 Januar 1902, Kolivand, S. 68). Bei diesen Beschreibungen verwendet er ziemlich generalisierende Ausdrücke wie: „so sind alle Perser“. Gleich nach dieser Missachtung vergleicht er den Menschen im Orient mit den Europäern. So kommt eine Dichotomie zustande, wo die Europäer anders als die fremde Orientalen sind, „[...] als ich ihnen (Perser) rundweg erklärte, dass wir Frengi (Europäer) uns zu einer Lüge nie herabwürdigen, waren sie anfangs sprachlos und begriffen offenbar nichts, wie ich in einer Lüge so etwas schlechtes sehen könne" (Brief an die Familie vom 16 Mai 1902, Kolivand, S. 136). In dieser Gegenüberstellung, während Mann über die Perser spricht, betont er tatsächlich die kulturelle Überlegenheit seiner eigenen Zivilisation gegenüber der orientalischen Welt. Andererseits definiert er sich selbst als Europäer. Daher können die Reisen als „Begegnung zwischen sich selbst und dem anderen" und eine „Verhandlung zwischen Ähnlichkeit und Unterschied" betrachtet werden (Thompson, 2011, S. 10).

Selbst wenn Mann über seinen Hund schreibt, zögert er nicht, eine Beziehung zur Sexualität der Iraner mit Hunden herzustellen.,[...] er (der Hund) behandelt das weibliche Geschlecht - wie die Perser - eben nur als Abzugskanal der überströmenden Lebenskräfte. Und das recht reichlich" (Brief an Friedrich Carl Andreas vom 29 April 1902, Kolivand, S. 121). Mann betrachtet die iranischen Volksgruppen als minderwertig. Deshalb stellt es für inn kein Problem dar, gemeinsame Aspekte mit Tieren zu finden. Um seine Beschreibungen und Beurteilungen, basierend auf negativen Stereotypen, über unterschiedlichen Volksgruppen in seiner inneren Welt zu legitimieren, sprach er nämlich von seinen eigenen Erfahrungen, die er auf iranischem und osmanischem Boden sammelte, denn er wurde von orientalischen Leuten belogen und betrogen.

In Manns Briefen tauchen immer wieder abwertende Bilder über das tägliche Leben auf. Die Diffamierung ihrer Esskultur bekommt auch ihren Anteil in seinen zahlreichen Briefen wie folgt: 
[...] aber der alte und seine schauderhaft hässliche dreckige Frau ${ }^{4}$ sich nun ans Zerlegen machten; ohne Gabel ohne Messer mit den Saufingern in das Fleisch hineinführen, da verging mir doch der Appetit. An so etwas muss ich mich erst langsam wieder gewöhnen. Ich war dicht am Gerben! (Brief an die Familie vom 20 Juni 1906, Kolivand, S. 505)

Das Essen mit den Fingern findet er barbarisch, ekelhaft und widerwärtig und vergleicht diese Tradition sogar mit dem Kannibalismus. Er hat nicht die Absicht zuzugeben, dass dies ein typisch zeitgenössisches Phänomen ist:

[...] man hätte tagtäglich am Essen teilnehmen und zusehen müssen, wie die Kerle wie die Kannibalen, ihren Braten mit den Fingern zerlegen, hätte Lehmwasser trinken müssen - alle solche freundlichen Einladungen abzulehnen, wäre fast Beleidigung gewesen. (Brief an die Familie vom 30 Juni 1906, Kolivand, S. 511)

Auch in einem Brief vom Bachtiaren-Land (Ardalan) beschreibt er seine Gefühle übertreibend am Abendessen folgendermaßen:

[...] die Leute mit den Fingern in dem fetten Reis umherarbeiten, die Fleischstücke vom Bratspiess herunterstreifen und alles mit der Hand in die hungrigen Mäuler stopfen, das anzusehen trägt auch gerade nicht zur Mehrung der Esslust bei. (Brief an die Familie vom 16 Mai 1902, Kolivand, S. 134).

Mann trennt den Orient vom Okzident mit präzisen und scharfen Linien und teilt eurozentristisch in zwei voneinander getrennte Kategorien. Im Vergleich zur europäischen Kultur erscheint der Orient in den Briefen als unterentwickelt, dreckig, barbarisch. Dass er nicht nur als Europäer, sondern auch als Stadtmensch die iranisch-kurdische Bevölkerung

4 Die europäischen Reisenden beschreiben die orientalischen Frauen mit bestimmten kulturellen Mustern und Stereotypen. Das Auffälligste an diesen Darstellungen besteht darin, dass die orientalischen Frauen unterdrückt, Polygamie und Gewalt ausgesetzt sind. Darüber hinaus wird die orientalische Frau im Allgemeinen mit generalisierender Hässlichkeit und Demütigung [Sinn] im Vergleich zu europäischen Frauen dargestellt. Diese als machtloses Wesen dargestellte Frau entspricht dem „orientalisierten“ Frauenbild im Europa des 19. Jahrhunderts. Mann war während ihrer iranischen und osmanischen Reisen aufgrund sozialer Traditionen nicht allzu sehr in die Frauenwelt involviert. Aus diesem Grund beschränkte sich seiner Wahrnehmung iranischer und kurdischer Frauen in seinen Briefen nur auf Darstellungen ihres äußeren Erscheinungsbildes, d.h. ihrer Hässlichkeit (Brief an die Familie vom 20 Juni 1906, Kolivand, S. 505, 244). Während die europäischen Reisenden sich mit den orientalischen Frauen in Verbindung setzen, dauert es nicht so lange, mit ihnen ins Gespräch zu kommen, um sie genau zu beschreiben. Aber trotzdem sind detaillierte Frauenbilder in Reiseberichten zu finden. 
in Provinzen beobachtet, verhindert geradezu ein Einfühlungsvermögen in das Leben im Orient.

Mann reiste in einen Ort - den Orient -, der beschrieben werden sollte. Das heißt, der Orient wird dargestellt, wie Reisende ihn wahrgenommen haben. Wie in Manns Fall, spielt die Verschmutzung des Orients eine zentrale Rolle bei seiner Urteilung. Er findet im Trinkwasser der Iraner auch etwas Abscheuliches.

[...] eine ekelhaft lehmige Flüssigkeit, die die Perser aber mit grosser Vorliebe trinken. Belehrt sie doch ihre Religion, dass jedes fliessende Wasser rein ist, welch merkwürdige Situationen aus dieser Lehre gelegentlich resultieren, will ich euch lieber nicht schreiben - ihr werdet sonst vielleicht auf einige Tage euren Appetit verlieren! (Brief an die Familie vom 16 Mai 1902, Kolivand, S. 137)

Überall im Orient sind es die Unordnung, die Verschmutzung und der Geruch der Stadt, welche auf den ersten Blick seine Aufmerksamkeit erregen. Er bringt immer wieder dieses wiederholende abwertende Ausdrücken zu Papier.

\begin{abstract}
[...] Muhammedaner, die dabei so dreckig sind, dass es einem hochkommt, wenn man die Kerls nur sieht. Selbst in einer Stadt, wie Sännändusch (Sanandadsch), starren alle Strassen vor Dreck. Jeglicher Hausabfall wird einfach auf die Strasse geworfen; dazu kommen dann die zahllosen Hunde-, Katzen- und Maultierleichen, die mitten in den Strassen der Stadt verwesen, und einen so ekligen Gestank verbreiten, dass man kaum zu athmen wagt. (Brief an die Familie vom 27 April 1903, Kolivand, S. 352)
\end{abstract}

Manns Beharren auf ihm auffallende Bemerkungen, die er abscheulich findet, seiner Familie zu übermitteln bringt die Gefahr mit sich, statt die objektive Einschätzung der fremden Außenwelt zu verzeichnen, eine negative Wertung darzulegen wie in diesem Beispiel (vgl. Hupfeld, 2007, S. 26).

Ich habe viel an schmutzt gesehen, aber soviel auf einen Haufen, wie hier in die Dschezire (Cizre), noch nirgend. Es ist einfach haarsträubend, und von den mannigfachen ekelhaften Gerüchen, die hier den Menschen gleich bösen Geistern umschweben, kann sich der in Berlin lebende Kulturmensch schwerlich eine auch nur der Wirklichkeit sich nährenden Vorstellung machen. Daher ist auch Dschezire 
als bösestes Fiebernest überall verschrieen. Ich werde so bald irgend möglich den Dreckstaub dieses Mistnestes von meinen Pantoffeln schütteln. (Brief an die Familie vom 28 September 1906, Kolivand, S. 559)

\section{Pauschalisierender Orient}

Auffällig ist, dass Mann üblicherweise pauschal von diversen Ethnien des Orients als Orientalen spricht. Was an Manns Haltung erinnert, sind Saids folgende Worte: „Es gibt Orientalen, Asiaten, Semiten, Muslime, Araber, Juden, Rassen, Mentalitäten, Nationen und dergleichen [...]" (Said, 1979, S. 154-155). In den Briefen spricht Mann über die Iraner, Kurden, Türken und ihre Untergruppierungen. Hierbei sieht Mann einerseits keine individuellen Unterschiede und beschränkt sich auf einige pauschale Urteile. Anderseits trennt er die orientalischen Volksgruppen voneinander ab mit differenzierten Stereotypen in ihrem Alltag. Seine Schilderungen über die Bachtiaren - ein Nomadenvolk im südwestlichen Iran - kann als konkretes Beispiel für differenzierte ethnische abwertende Stereotype genannt werden.

\section{[...] die Bakhtiaren sind eine ganz besondere edle Rassen von Persern. Was ich von diesen Leuten angetroffen habe, war noch fauler, diebischer und verschmitzter, jedenfalls aber unglaublich viel unfreundlicher als alle anderen Perser. Das gewöhnliche Volk lebt wie das liebe Vieh. Das gilt ja vielleicht von allen Nomaden ist. (Brief an die Familie vom 25 Mai 1902, Kolivand, S. 141)}

Die Darstellung der orientalischen Gesellschaften mit ihren besonderen, differenzierten und gemeinsamen Attributen sowie Traditionen, Religionen und Sprachen sind seit dem 16. Jahrhundert mit den europäischen Reiseberichten verflochten. In diesem Zusammenhang kann man sagen, dass die Reiseliteratur zwar eine wichtige Quelle für wissenschaftliche Daten in ethnographisch-anthropologischen Studien ist (vgl. Rubie's 2006, S. 242), sie jedoch häufig ein Mittel war, um die orientalischen Gesellschaften in ethnozentrische kulturelle Muster einzusperren.

Das hegemonial-koloniale Gedächtnis spielt eine wichtige Rolle bei der Rechtfertigung der abwertenden Stereotype wie Unsauberkeit, Faulheit, Verlogenheit und Wildheit. Im kolonialen Amerika war es zum Beispiel ein systematischer Versuch, einen Indianer als „Entführer, Kindermörder und Kopfhaut-Sammler darzustellen, als Apologie für die Brutalität der Weißen“ (Kabbani, 1986, S. 4). Mann übernimmt und praktiziert in seinem 
Studiengebiet dieses Gedächtnis mit „unerschütterlichen abstrakten Maximen über die Zivilisation" (Said 1979, S. 52) in seiner Reise, ohne zu versuchen, sich in die bereisten Gesellschaften hineinzuversetzen. Die direkte Begegnung mit dem orientalischen Fremden reicht nicht aus, um bei seiner Darstellung starke ethnische Stereotype zu durchbrechen. Mann ist ein Orientalist und Reisender, der Menschentypen aussucht, die zu seinen übernommenen Stereotypen passen. Die zahlreichen anderen Gruppen innerhalb der orientalischen Gesellschaft, die nicht seinen Stereotypen entsprachen, sind in den Briefen kaum zu finden.

Über Bachtiaren schreibt er weiter: „[...] Versprechen nicht halten, Lügen und Betrügen wird anscheinend auch unter Bachtiaren zu den guten Witzchen gerechnet. Ob die vornehmeren Klassen, die Khanfamilien, besser sind, muss ich erst noch sehen" (Brief an die Familie vom 25 Mai 1902, Kolivand, S. 142). Obwohl er von einer beliebten Bachtiaren Familie gastlich und freundlich aufgenommen wird, spricht er trotzdem von ihnen auf abwertende Weise. Manns feste Vorurteile gegenüber den orientalischen Volksgruppen sind das größte Hindernis, den fremden Orientalen zu glauben. Er beschreibt dies in seinen Briefen wie folgt: „[...] sind ja auch die großartigen Versprechungen des Herrn İlbegi, wie ihr ja auch gesehen habt, wie eine Seifenblase gewesen: Nichts als Luft dahinter." An seinem nächsten Haltepunkt - in Malamir - hofft er die Gelegenheit zu haben, seine „Anschauung vom Wesen der Bakhtiaren zu ändern" (Brief an die Familie vom 25 Mai 1902, Kolivand, S. 142), dazu ist er aber nicht in der Lage. Während seiner Reise verzichtet er niemals darauf, die orientalischen Volksgruppen pauschalisierend zu beschreiben. Mann versucht, die Beziehung der orientalischen Menschen zur Lüge in ihrer eigenen Sprache zu vermitteln: „[... ] der Khan war ein drolliger Kauz. Er sagte, dass er wahrscheinlich mehrere Jahre in Europa diesmal zubringen werde. Jeder Mensch, der frei ist, braucht nicht zu lügen; aber wir, die wir unfrei sind, müssen lügen. So muss ich z.B. dem Schach vorlügen, ich sei krank. Sonst lässt er mich nicht nach Europa schicken" (Brief an die Familie vom 6 Juni 1902, Kolivand, S. 153). Er möchte hier durch Khans eigene Äußerungen mit einem hegemonial-kolonialen Stil betonen, dass der Orientale tatsächlich nicht frei ist.

\section{Fazit}

Das Thema dieser Studie war die Fremdwahrnehmung und Darstellung von Oskar Mann. Er empfand in seinen Reisebriefen, die das Textkorpus der vorliegenden Arbeit bilden, den Orient als Raum und den orientalischen Mensch als fremd. Unterschiedliche 
Orientvorstellungen und -wahrnehmungen formten Manns Eindrücke. Das heißt, die Repräsentation einer fremden Kultur kann nicht unabhängig von der Ideologie des Reisenden sein. Obwohl die Orientreisenden während ihrer Reise Wissen für verschiedene Disziplinen, wie zum Beispiel orientalische Philologie, Kulturwissenschaft, Ethnologie, Anthropologie usw. produzieren und es transferieren, können die Reisen ebenfalls als kulturelle Begegnungen betrachtet werden, da die Reisenden sich die verschiedenen Volksgruppen begegnen. Bei diesem Zusammentreffen beschreiben die Reisenden die einheimischen Gesellschaften des bereisten Landes sehr detailliert, mit subjektiver und eigenkultureller Sicht. Einerseits machte Mann Forschungen über die iranischen Sprachen und Dialekte, andererseits produzierte er in seinen Reisebriefen Bilder und Stereotypen mit bestimmten kulturellen Mustern über die unterschiedlichen Volksgruppen im Iran und im Osmanischen Reich. Mann, als ein Iranist, beschäftigte sich zwar jahrelang mit den Sprachen und Dialekten dieser Zivilisation, er begegnete aber den iranischen Volksgruppen kulturell nicht mit Sympathie und Begeisterung. Die folgenden Aspekte beeinflussten Manns stereotype Darstellungen und Antipathie von den Menschen im Orient: i) Er begann seine Reise mit einigen in Europa weit verbreiteten generalisierenden Ressentiments über den Orient, die er ohne zu hinterfragen übernahm, wie beispielsweise Unsauberkeit, Faulheit, Verlogenheit, und Wildheit. ii) Er nimmt den Orient mit traditionelleurozentrischer und ethnozentrischer Selbstgewissheit wahr iii) und vermittelt es in seinen Briefen in einem anti-islamischen Ton. iv) Er befindet sich unter den orientalischen Menschen mit einer hegemonial-kolonialen Denkweise. Dies veranlasste ihn dazu, die Volksgruppen des bereisten Landes auf abwertende Weise darzustellen.

Begutachtung: Extern begutachtet.

Interessenkonflikt: Es besteht kein Interessenkonflikt.

Finanzielle Förderung: Dieser Beitrag wurde von keiner Institution finanziell unterstützt.

Peer-review: Externally peer-reviewed.

Conflict of Interest: The author has no conflict of interest to declare.

Grant Support: The author declared that this study has received no financial support.

\section{Literaturverzeichnis}

Avcı, R. (2020). Seyahat Notları ve Oryantalist Bilgi Üretimi: Eduard Sachau (1845-1930) Örneği. Diyalog Interkulturelle Zeitschrift Für Germanistik, 8(1), 29-47.

Avcı, R. (2021). Oryantalist Oskar Mann'ın (1867-1917) Osmanlı Seyahati Mektuplarında Zazacanın Tasnifi. Nüsha, 21(52), 161-178. 
Bies, M.-Košenina, A. (2014). Reisen und Wissen. Einleitung. Zeitschrift für Germanistik, 24(1), 7-9.

Brenner, P. J. (1990). Der Reisebericht in der deutschen Literatur: Ein Forschungsüberblick als Vorstudie zu einer Gattungsgeschichte. Tübingen: Max Niemeyer Verlag.

Edwards, J., \& Graulund, R. (2011). Introduction: Reading Postcolonial Travel Writing. Justin (Hrgs.) Edwards/Rune Graulund. Postcolonial Travel Writing Critical Explorations. Chippenham and Eastbourne: CPI Antony Rowe. 1-17: 1-16.

Fendri, M. (1996). Kulturmensch in »barbarischer« Frem-de. Deutsche Reisende im Tunesien des 19. Jahrhunderts. München: ludicium.

Funke, H. G. (1982). Studien zur Reiseutopie der Frühaufklärung: Fontenelles Histoire des Ajaoiens. Heidelberg: Carl Winter Universitätsverlag.

Gogiaschwili, E. (2008). Das Stereotyp der Georgierin in der Abendländischen Reiseliteratur vom 17.-19. Jahrhundert, Saeculum. Jahrbuch für Universalgeschichte, 59(2), 253-268.

Hentsch, T. (2008). Hayali Doğu: Batı́nın Akdenizli Doğu'ya Politik Bakışı. İstanbul: Metis Yayıncılık.

Huff, D. Archeological Excavations and Studies, The Encyclopædia Iranica, https://iranicaonline.org/articles/ germany-ii (letzter Zugriff: 01. 31. 2021).

Hupfeld, T. (2007). Zur Wahrnehmung und Darstellung des Fremden in ausgewählten französischen Reiseberichten des 16. bis 18. Jahrhunderts."Il les faut voir et visiter en leur pays". Göttingen: Universitätsverlag Göttingen.

Kabbani, R. (1986). Europe's Myths of Orient. Hampshire-London: The Mcmillian Press.

Kolivand, M. (2014). Persische und kurdische Reiseberichte Die Briefe des Berliner Orientalisten Oskar Mann während seiner beiden Expeditionen in den Vorderen Orient 1901-1907. Harrassowitz: Wiesbaden.

Lazaare, K. (1998). Marokko in deutschen Reiseberichten des 19. und beginnenden 20. Jahrhunderts. Frankfurt am Main: Peter Lang Verlag.

Lemke, B.- Rosbeiani, P. (2018). Unternehmen Mammut. Ein Kommandoeinsatz der Wehrmacht im Nordirak 1943. Bremen: Edition Falkenberg.

Mann, O. (1891). Abu'l-Hasan Ibn-Muhammad Āmīn: Das Muǰmilet-târikh-i ba'dnâdirîje des Ibn Muhammed Emîn Abu'I-Hasan aus Gulistâne. Leiden: Brill.

Mann, O. (1903). Archäologisches aus Persien. Globus, 83, 327-331.

Mann, O. (1906). Die Mundart der Mukri-Kurden. Berlin: Druck und Verlag von Georg Reimer.

Mann, O. (1910). Die Tâjîk-Mundarten der Provinz Fârs. Berlin: Georg Reimer.

Marchand, S. (2009). German Orientalism in the Age of Empire Religion, Race, and Scholarships. New York: Cambridge University Press.

Osterhammel, J. (1989). Distanzerfahrung: Darstellungsweisen des Fremden im 18. Jahrhundert. In. H.-J. König (Hrsg.). Der europäische Beobachter außereuropäischer Kulturen: Zur Problematik der Wirklichkeitswahrnehmung. Berlin: Duncker \& Humblot: 9(42).

Osterhammel, J. (1989). Reisen an die Grenzen der Alten Welt : Asien im Reisebericht des 17. und 18. Jahrhunderts. In. Peter Jürgen Brenner (Hrsg.). Der Reisebericht: die Entwicklung einer Gattung in der deutschen Literatur. Frankfurt a.M.: Suhrkamp: 224-260. 
Paul, L. (2020). Karl Hadank (1882-1945) and the Kurdisch-Persiche Forschungen: Ambitions, Achievements and the ideological Entanglements. Diyâr Zeitschrift für Osmanistik, Türkei- und Nahostforschung, 29, 289-309.

Rubie's, J. P. (2006). Travel writing and ethnography. In. Peter Hulme-Tim Youngs (Hrsg.). The Cambridge Companion to Travel Writing. Cambridge: Cambridge University Press: 242-260.

Said, E. (1979). Orientalism. New York: Vintage Books.

Soukah, Z. (2016). Der Orient als kulturelle Selbsterfindung der Deutschen, (unveröffentliche Dissertation) Philosophische Fakultät der Heinrich-Heine-Universität Düsseldorf.

Teilhaber, A. (2020). Friedrich Rosen: Orientalist Scholarship and International Politics. Berlin: De Gruyter.

Thompson, C. (2011). Travel Writing. London: Routledge.

Voltrova, M. (2010). Zu imagologischen Interpretationsverfahren-eine methodenkritische Anmerkung. Germanoslavica, 1-2(21), 135-145.

Voss, K. (2001). Amerikanisierung.Amerikanisierung?-Die USA in der Berichterstattung zum Bundestagswahlkampf 1998. In. Hans J. Kleinsteuber (Hrsg.). Aktuelle Medientrends in den USA: Journalismus, politische Kommunikation und Medien im Zeitalter der Digitalisierung. Wiesbaden: Springer Verlag: 252-264.

Wagner, V. (2014). Mehmed Rauf'un Avrupa Seyahatnamesi Üzerine Bir Değerlendirme. Güney-Doğu Avrupa Araştırmaları Dergisi, 2(26), 97-127.

Wagner, V. (2016). Imagologie der Fremde. Das Londonbild eines osmanischen Reisenden (Göttingen: Bonn University Press. 
